\title{
Estimation of Surface Breakdown Voltage of Solid/Gas Composite Insulation with Embedded Electrode
}

\author{
Takahiko Yamashita, Kazuhisa Iwanaga, Tomohiro Furusato, \\ Hiroyuki Koreeda, Tomoyuki Fujishima \\ Nagasaki University \\ Graduate School of Engineering \\ Nagasaki 852-8521, Japan
}

Naoki Asari and Junichi Sato

Toshiba Corporation Power Systems Company

1, Toshiba-cho, Fuchu-shi

Tokyo 183-8511, Japan

\begin{abstract}
Solid insulated switchgear (SIS) has been developed as a substitution for medium voltage $\mathrm{SF}_{6}$ gas insulated switchgear. Its main circuits including a vacuum interrupter are coated with epoxy resin, therefore, a solid/gas insulation system is formed. For further miniaturization and higher stress design of SIS, the authors investigated on the surface insulation of the solid/gas insulation system containing an embedded electrode. In the present study, the surface breakdown voltage was estimated based on the surface discharge propagation characteristics. From the measurement of the surface flashover voltage on a dielectric against the surface distance, the relation between the surface flashover voltage and the surface distance was found to be expressed with an equation derived from the empirical equation presented by Toepler. Then, the surface breakdown voltage was estimated based on the obtained equation. The estimated values were compared with the experimental data obtained varying a parameter of the radius of the embedded electrode, thickness of the epoxy resin plate or the radius of the plate. As a result, a good correlation was obtained. Therefore, the estimation method of the surface breakdown voltage on the solid/gas insulation with embedded electrode was established.
\end{abstract}

Index Terms - Composite insulation, electric breakdown, estimation, surface discharge, switchgear.

\section{INTRODUCTION}

IN an electric power system, switchgear is used for changing circuit connections. It also takes the role of isolating a fault when a problem occurs in the system. In that case, switchgear operates so that it isolates the fault within a minimum area and prevents a spread of the problem towards important equipment. Therefore, high reliability in operation is required for the switchgear.

Recently, most of the medium voltage switchgear is cubicle-type low pressure $\mathrm{SF}_{6}$ gas insulated switchgear. However, $\mathrm{SF}_{6}$ gas was designated as one of the targets of emission control in the global warming prevention conference held in 1997. Then, research and development of $\mathrm{SF}_{6}$ gas free systems were actively advanced [1,2].

Regarding the situation mentioned above, a solid insulated switchgear (SIS) has been developed [2]. The main circuits of

Manuscript received on 4 January 2016, in final form 30 May 2016, accepted 30 May 2016.
SIS including a vacuum interrupter are coated with epoxy resin. However, improvement of the surface insulation performance of the solid/gas composite insulation system is necessary for the development of higher stress and compact design.

Therefore, the authors investigated on the surface insulation of the solid/gas composite insulation system. In the previous study [3], metal electrode was embedded into the insulation material of epoxy resin and its effect on the insulation performance was investigated. In the present study, the surface discharge propagation characteristics were measured and the characteristics were found to be expressed with an equation derived from the empirical equation presented by Toepler. Then, the surface breakdown voltage was estimated based on the obtained equation. The estimated values were compared with the experimental data obtained by varying a parameter of the radius of the embedded electrode, thickness of the epoxy resin plate or the radius of the plate. As a result, a good 
correlation was obtained. Therefore, the estimation method of the surface breakdown voltage on the solid/gas insulation system with embedded electrode was established.

\section{SURFACE BREAKDOWN MODEL}

Figure 1 shows the cross section of a test sample of the solid/gas composite insulation system. This test sample was the model of insulation system of SIS and was composed of a disc-shaped epoxy resin plate with a back-side electrode and a disc-shaped embedded electrode. The back-side electrode was formed by painting a conductive layer on the underside of the insulation plate. The embedded electrode was made of copper with a thickness of $2 \mathrm{~mm}$. It was rounded at the edge with a radius of curvature of $1 \mathrm{~mm}$. The separation between the bottom of the embedded electrode and the back-side electrode was $4 \mathrm{~mm}$. The embedded electrode was connected to the center rod electrode which was made of copper with a radius of $5 \mathrm{~mm}$.

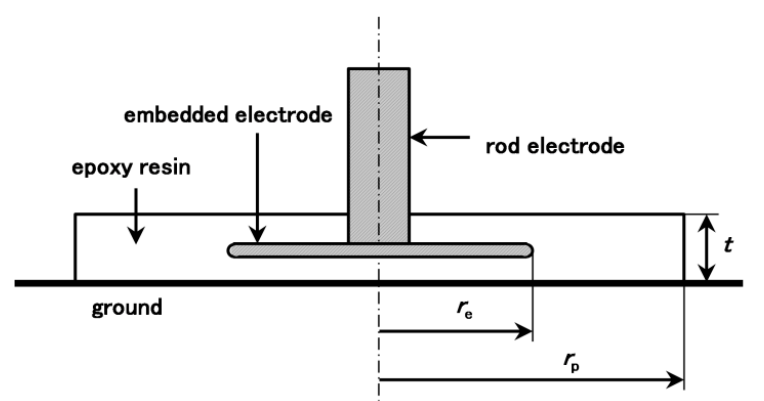

Figure 1. A model of composite insulation system with embedded electrode. $r_{\mathrm{p}}$ is the radius of the disc-type epoxy resin plate, $r_{\mathrm{e}}$ is the radius of the embedded electrode and $t$ is the thickness of the epoxy resin plate [3].

In the previous study [3], the authors investigated on the surface breakdown voltage (BDV) and the partial discharge inception voltage (PDIV) by varying the radius of the embedded electrode $\left(r_{\mathrm{e}}\right)$, thickness of the disc-type epoxy resin plate $(t)$ or the radius of the epoxy resin plate $\left(r_{\mathrm{p}}\right)$ under ac or impulse voltage application. As a result, it was found that the surface discharge initiated at the point of the maximum electric field strength along the surface and it propagated in both directions towards the center rod electrode and towards the ground electrode. The surface breakdown is supposed to be caused when both of the surface discharges reach the opposite electrodes. However, it is slightly complicated. The polarity of the surface discharge propagates on each surface is different. The electrostatic property of each surface is also different. Moreover, the applied voltage is divided into both surfaces.

In order to simplify the discussion, the authors introduce an imaginary electrode at the point of the maximum field strength. As shown in Figure 2, the surface can be separated into two parts according to the direction of the surface discharge propagation. If the polarity of the applied voltage is positive, a positive surface discharge propagates from the point of maximum field strength towards the ground and a negative one propagates from the point towards the center rod electrode. The electrostatic property of each surface is different, because the thickness of the epoxy resin is not the same.
Now, the authors introduce a hypothesis for the model of the surface breakdown process as follows.

(a) If one of the surface discharges reaches the opposite electrode as shown in Figure $2 \mathrm{~b}$ or $2 \mathrm{c}$, the surface flashes over and all of the voltage is applied to the other surface.

(b) Following (a), surface breakdown is accomplished if the applied voltage is high enough to flash over the other surface.

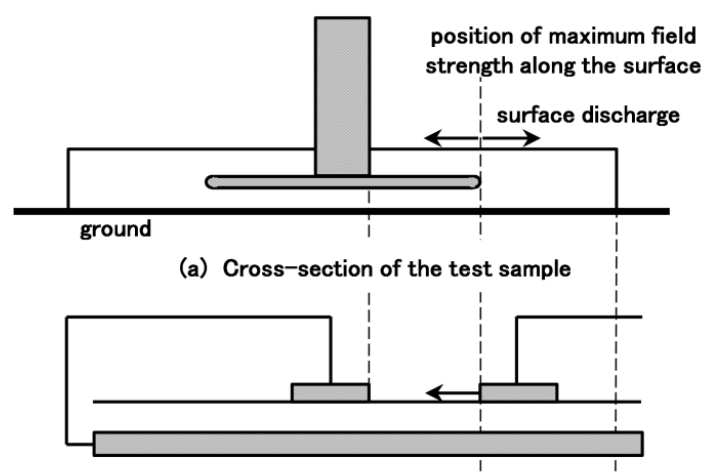

(b) Surface composition between the center electrode and the position of the maximum field strength

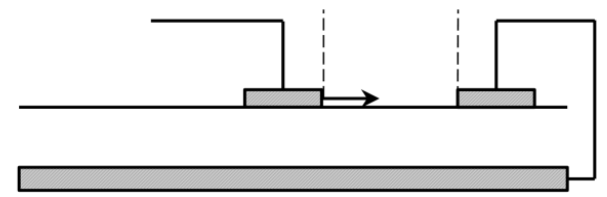

(c) Surface composition between the position of the maximum field strength and the ground

Figure 2. A separated surface model of composite insulation system with embedded electrode.

\section{BDV ESTIMATION PROCEDURE}

From the previous section, BDV of the test sample can be estimated based on the flashover characteristics of each surface in Figure 2. As already known widely, the surface flashover characteristics depend on the applied voltage and the distributed electrostatic capacity per unit area of a dielectric [4, 5]. However, the characteristics are slightly different among the researchers. In the present study, the characteristics were measured using disc-type epoxy resin plates experimentally. For BDV estimation the potential at the point of the maximum field strength is necessary. The potential was calculated using Ansoft Maxwell (Ansoft Japan Co., Ltd.) based on finite element method.

\subsection{SURFACE FLASHOVER CHARACTERISTICS ON DIELECTRIC}

The arrangement of electrodes and a dielectric for the measurement of the surface flashover characteristics is shown in Figure 3. Surface flashover characteristics were measured with disc-type epoxy resin plates without embedded electrode.

The specifications of the disc-type epoxy resin plates are shown in Table 1. The surface flashover characteristics were measured under impulse voltage application.

There are several equations which expressed the surface discharge propagation characteristics on a dielectric. Equation 


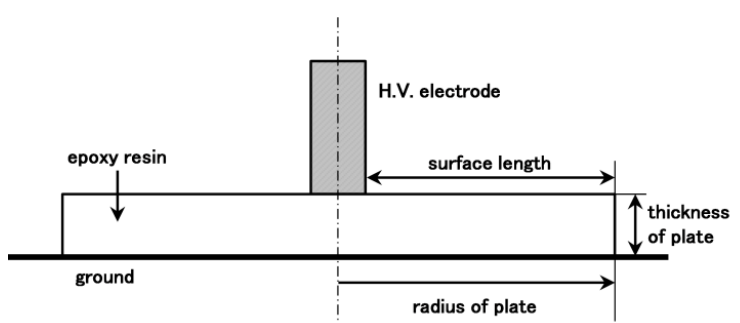

Figure 3. Electrode arrangement for the measurement of the surface flashover characteristics.

Table 1. Specifications of test samples used for the examination of the surface discharge propagation characteristics.

\begin{tabular}{c|c|c}
\hline $\begin{array}{l}\text { Radius of the } \\
\text { plate }(\mathrm{mm})\end{array}$ & $\begin{array}{l}\text { Thickness of the } \\
\text { plate }(\mathrm{mm})\end{array}$ & $\begin{array}{c}\text { Distributed } \\
\text { electrostatic } \\
\text { capacity per unit } \\
\text { area }\left(\mathrm{F} / \mathrm{m}^{2}\right)\end{array}$ \\
\hline 50 & 11 & $2.737 \times 10^{-9}$ \\
100 & 9 & $3.345 \times 10^{-9}$ \\
100 & 11 & $2.737 \times 10^{-9}$ \\
100 & 15 & $2.007 \times 10^{-9}$ \\
100 & 21 & $1.434 \times 10^{-9}$ \\
250 & 11 & $2.737 \times 10^{-9}$ \\
\hline
\end{tabular}

(1) is one of the equations which is an empirical equation presented by Toepler [4].

$$
l_{\mathrm{g}}=\mathrm{K}_{\mathrm{a}} V_{\mathrm{g}}^{4} C_{\mathrm{a}}^{\frac{3}{2}}
$$

where $l_{\mathrm{g}}(\mathrm{cm})$ is the surface discharge length, $V_{\mathrm{g}}(\mathrm{kV})$ is the applied voltage in peak value, $C_{\mathrm{a}}\left(\mathrm{pF} / \mathrm{cm}^{2}\right)$ is the distributed electrostatic capacity per unit area and $\mathrm{K}_{\mathrm{a}}$ is a constant. Toepler gave $\mathrm{K}_{\mathrm{a}}=3.4 \times 10^{-5}$ for positive polarity.

From equation (1), the surface flashover voltage can be derived if we assume that the surface flashover takes place when the surface discharge reaches the opposite electrode. Then, the surface flashover voltage $V(\mathrm{~V})$ in peak value can be expressed as equation (2)

$$
V=\mathrm{K}_{\mathrm{b}} l^{\frac{1}{4}} C_{\mathrm{b}}^{-\frac{3}{8}}
$$

where $l(\mathrm{~m})$ is the surface length, $C_{\mathrm{b}}\left(\mathrm{F} / \mathrm{m}^{2}\right)$ is the distributed electrostatic capacity per unit area and $\mathrm{K}_{\mathrm{b}}$ is a constant which is derived from $\mathrm{K}_{\mathrm{a}}$ and is $\mathbf{7 3 . 6}$ for positive polarity.

Equation (2) can be used for both ac and impulse surface flashovers. However, ac surface flashover voltage is known to be affected by the conditions such as the surface resistance. Furthermore, the equations expressed the surface flashover characteristics are different among the researchers. Therefore, the relation between surface distance and surface flashover voltage was measured under impulse voltage in the present study.

The surface flashover voltage was measured with $1.2 / 50 \mu \mathrm{s}$ impulse voltage and voltage elevation method. Voltage elevation was started from around $80 \%$ of the estimated flashover voltage and the step was about $1 \mathrm{kV}$. Measurement was done more than 20 times for each condition of samples listed in Table 1 and the minimum value was employed as the measured flashover voltage. In the case of the measurement under impulse voltage application, the residual charge of former discharge may influence on the surface flashover voltage. Consequently, the residual charge was removed before each voltage application. The influence was also examined without charge removal. The influence was revealed to vary with the kind of the applied voltage and the polarity in the case of impulse.

Figure 4 shows the relation between the surface flashover voltage and the surface distance. Figure 5 shows the relation between the surface flashover voltage and the thickness of the insulation plate. According to the variation of the thickness of the plate, the distributed electrostatic capacity per unit area varies as shown in Table 1. From these figures, the measured surface flashover voltages were found to be lower than the calculated values using equation (2). However, the variation of the flashover voltage was similar to the calculated values using equation (2).

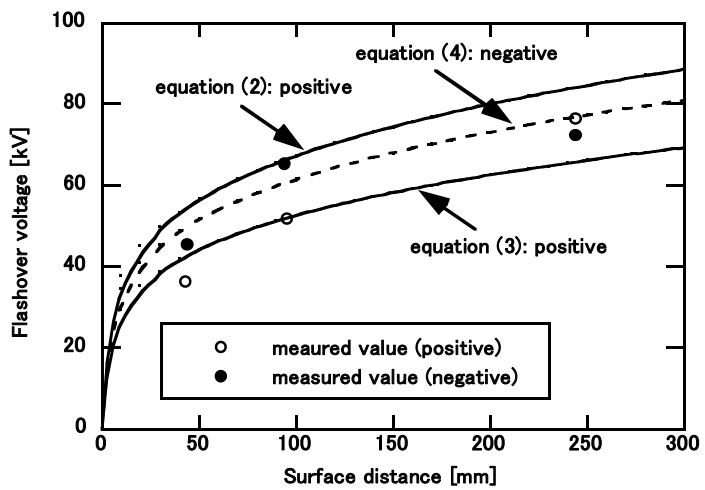

Figure 4. Relation between surface flashover voltage and the surface distance (thickness of the plate was $11 \mathrm{~mm}$ ).

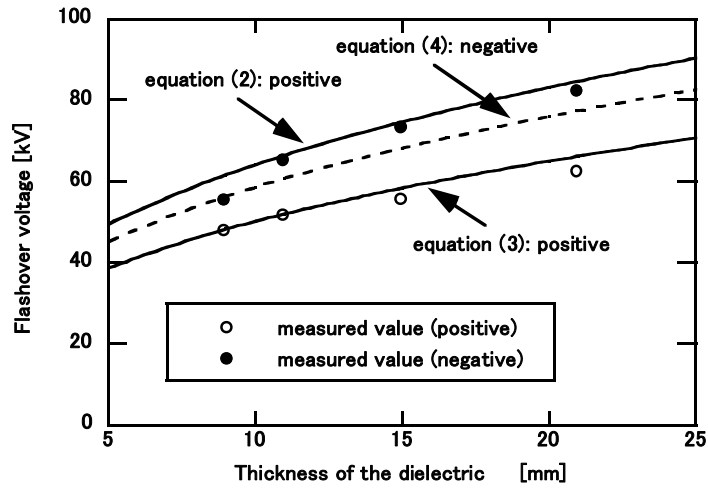

Figure 5. Relation between surface flashover voltage and the thickness of the dielectric (radius of the plate was $100 \mathrm{~mm}$ ).

Then, the authors assumed that the surface flashover voltage $V$ is proportional to $l^{\frac{1}{4}} C_{\mathrm{b}}^{-\frac{3}{8}}$ as in equation (2), and the authors determined the values of $K_{b}$ from the measured data with least squares method. It was 57.56 for positive and 67.25 for negative, respectively. Therefore, equation (2) was expressed in the following equations (3) and (4) which were used for the estimation of surface BDV. These equations are shown in Figures 4 and 5. 


$$
\begin{gathered}
V_{\text {pos. }}=57.56 l^{\frac{1}{4}} C_{\mathrm{b}}^{-\frac{3}{8}} \\
V_{\text {neg. }}=67.25 l^{\frac{1}{4}} C_{\mathrm{b}}^{-\frac{3}{8}}
\end{gathered}
$$

\subsection{BDV ESTIMATION PROCEDURE}

Firstly, we have to take the voltages across both surfaces, inner and outer into consideration. The divided voltages of inner and outer surfaces are expressed as follows

$$
\begin{aligned}
& V_{\text {out. }}=\mathrm{D} V_{\mathrm{a}} \\
& V_{\text {in. }}=(1-\mathrm{D}) V_{\mathrm{a}}
\end{aligned}
$$

where $V_{\text {in. }}$ and $V_{\text {out. }}$ are the voltages applied inner and outer surfaces when the applied voltage is $V_{\mathrm{a}}$. $\mathrm{D}(0 \leq \mathrm{D} \leq 1)$ is the divided voltage ratio and $\mathrm{D} V_{\mathrm{a}}$ also means the potential of the point of the maximum field strength to the ground. The potential was calculated using Ansoft Maxwell (Ansoft Japan Co., Ltd.). The obtained values of $\mathrm{D}$ will be shown in Table 2 (Section 4).

Secondly, we have to think about which surface flashes over first. The applied voltage when either surface flashes over is necessary. Substituting the surface flashover voltage for $V_{\text {in. }}$ or $V_{\text {out. }}$, the applied voltage when either surface flashes over can be obtained. However, the polarity of the applied voltage and the polarity of the surface discharge should be taken into consideration. There are three cases which are characterized according to the kind of the voltage applied to the center rod electrode and the polarity of the applied voltage.

Note that the thickness of inner and outer surfaces are different and the distributed electrostatic capacity per unit area is also different because $C_{\mathrm{b}}$ varies with the thickness of the dielectric. In the following equations, $C_{\mathrm{b}}=C_{\mathrm{b}}^{\prime}$ means that the thickness is different from the thickness of the plate. $l_{\text {in. }}$ and $l_{\text {out. }}$ mean the lengths of the inner and outer surfaces, respectively.

(a) Positive impulse voltage application

$V_{\text {pos. }}$ and $V_{\text {neg. }}$ should be substituted for $V_{\text {out. }}$ and $V_{\text {in. }}$, respectively.

$$
\begin{aligned}
& V_{\mathrm{F}_{\text {in. }}}=\left(\frac{1}{1-\mathrm{D}}\right) V_{\text {neg. }} \quad\left(C_{\mathrm{b}}=C_{\mathrm{b}}^{\prime}, l=l_{\text {in. }}\right) \\
& V_{\mathrm{F}_{\text {out. }}}=\frac{1}{\mathrm{D}} V_{\text {pos. }} \quad\left(l=l_{\text {out. }}\right)
\end{aligned}
$$

where $V_{\mathrm{F}_{\text {in }}}$ and $V_{\mathrm{F}_{\text {out. }}}$ are the peak values of the applied voltage when inner and outer surfaces flash over, respectively.

(b) Negative impulse voltage application

$V_{\text {neg. }}$ and $V_{\text {pos. }}$ should be substituted for $V_{\text {out. }}$ and $V_{\text {in. }}$, respectively.

$$
\begin{aligned}
& V_{\mathrm{F}_{\text {in. }}}=\left(\frac{1}{1-\mathrm{D}}\right) V_{\text {pos. }} \quad\left(C_{\mathrm{b}}=C_{\mathrm{b}}^{\prime}, l=l_{\text {in. }}\right) \\
& V_{\mathrm{F}_{\text {out. }}}=\frac{1}{\mathrm{D}} V_{\text {neg. }} \quad\left(l=l_{\text {out. }}\right)
\end{aligned}
$$

(c) Ac voltage application

The polarity of the surface discharge changes alternatively and the flashover voltage of positive polarity is lower than that of negative. Therefore, $V_{\text {pos. }}$ should be substituted for $V_{\text {in. }}$ and $V_{\text {out. }}$. Note that all of the voltages are expressed in peak values.

$$
\begin{aligned}
& V_{\mathrm{F}_{\text {in. }}}=\left(\frac{1}{1-\mathrm{D}}\right) V_{\text {pos. }} \quad\left(C_{\mathrm{b}}=C_{\mathrm{b}}^{\prime}, l=l_{\text {in. }}\right) \\
& V_{\mathrm{F}_{\text {out. }}}=\frac{1}{\mathrm{D}} V_{\text {pos. }} \quad\left(l=l_{\text {out. }}\right)
\end{aligned}
$$

From the comparison between $V_{\mathrm{F}_{\text {in. }}}$ and $V_{\mathrm{F}_{\text {out. }}}$, which surface flashes over first can be revealed.

Finally, we have to compare the applied voltage when either surface flashes over first with the flashover voltage of un-shortened surface. As all of the applied voltage is assumed to be applied to the un-shortened surface, the surface breakdown takes place when the applied voltage is higher than the flashover voltage of un-shortened surface. On the other hand, if the applied voltage is lower than the flashover voltage of un-shortened surface, the flashover voltage of un-shortened surface becomes the surface breakdown voltage.

As it is slightly complicated, an example is introduced. Figure 6 shows an example of the calculated results in the case $t=11(\mathrm{~mm}), r_{\mathrm{p}}=100(\mathrm{~mm}), r_{\mathrm{e}}$ is varied and negative impulse voltage is applied to the center rod electrode.

In the case where $r_{\mathrm{e}}$ is $5 \mathrm{~mm}$, surface breakdown voltage, $\mathrm{BDV}$ is determined from the negative surface flashover voltage of the epoxy resin plate with the surface distance of 95 $\mathrm{mm}$ and the thickness of $11 \mathrm{~mm}$

$$
C_{\mathrm{b}}=\frac{\varepsilon_{\mathrm{r}} \times 8.854 \times 10^{-12}}{d}
$$

where $\varepsilon_{\mathrm{r}}$ is the relative permittivity of 3.4 , and $d$ is the thickness of dielectric and is the same as the thickness of the plate of $11 \times 10^{-3} \mathrm{~m}$, under the present condition. Then, $C_{\mathrm{b}}$ becomes $2.737 \times 10^{-9} \mathrm{~F} / \mathrm{m}^{2}$. BDV is determined as follows.

$$
\begin{aligned}
& \mathrm{BDV}=V_{\mathrm{F}_{\text {out. }}}=\frac{1}{\mathrm{D}} V_{\text {neg. }} \\
& \left(C_{\mathrm{b}}=2.737 \times 10^{-9}, l=95 \times 10^{-3}, \mathrm{D}=1\right)
\end{aligned}
$$

When $r_{\mathrm{e}}$ is larger than $5 \mathrm{~mm}$, the thickness $d$ of inner side becomes $5 \times 10^{-3} \mathrm{~m}$, because the thickness of the epoxy resin plate is $11 \mathrm{~mm}$, the thickness of the embedded electrode is 2 $\mathrm{mm}$ and the distance between the embedded plate and the back-side electrode is $4 \mathrm{~mm}$. Then, $C_{\mathrm{b}}$ becomes $6.021 \times 10^{-9}$ $\mathrm{F} / \mathrm{m}^{2}$. However, $V_{\mathrm{F}_{\text {in. }}}$ and $V_{\mathrm{F}_{\text {out }}}$ was calculated except for the small $r_{\mathrm{e}}$ region. In the case where $5 \leq r_{\mathrm{e}} \leq 10(\mathrm{~mm})$, the point of the maximum field strength appears at the surface of the center rod electrode. Then, BDV cannot be estimated according to the procedure mentioned above. The authors suppose that BDV in this region is almost the same as the case where $r_{\mathrm{e}}=5(\mathrm{~mm})$. The particularity in this region will be explained again in DISCUSSION.

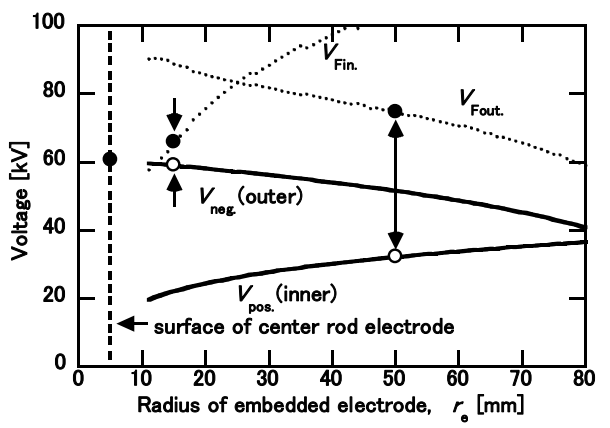

Figure 6. An example of surface flashover characteristics under negative impulse application. $t=11(\mathrm{~mm})$ and $r_{\mathrm{p}}=100(\mathrm{~mm})$. 
When $r_{\mathrm{e}}$ is $15 \mathrm{~mm}, V_{\mathrm{F}_{\text {in }}}$ is lower than $V_{\mathrm{F}_{\text {out. }}}$ that means the inner surface flashes over first. Then, BDV is obtained from the comparison between $V_{\mathrm{F}_{\text {in. }} \text {. }}$ and the surface flashover voltage of the outer surface $\left(V_{\text {neg. }}\right) . V_{\mathrm{F}_{\text {in. }}}$ is higher than $V_{\text {neg. }}$, then $V_{\mathrm{F}_{\text {in }}}$. becomes BDV.

$$
\begin{aligned}
& \mathrm{BDV}=V_{\mathrm{F}_{\text {in. }}}=\left(\frac{1}{1-\mathrm{D}}\right) V_{\text {pos. }} \\
& \left(C_{\mathrm{b}}=C_{\mathrm{b}}^{\prime}=6.021 \times 10^{-9}, l=15 \times 10^{-3}\right)
\end{aligned}
$$

On the other hand, when $r_{\mathrm{e}}$ is larger than $25 \mathrm{~mm}\left(r_{\mathrm{e}}=50\right.$ (mm) for example), $V_{\mathrm{F}_{\text {out }}}$ is lower than $V_{\mathrm{F}_{\mathrm{in}} \text {. }}$ that means the outer surface flashes over first. Then, BDV is obtained from the comparison between $V_{\mathrm{F}_{\text {out }}}$ and the surface flashover voltage of the inner surface $\left(V_{\text {pos. }}\right.$ where $C_{\mathrm{b}}$ is $6.021 \times 10^{-9}$ $\left.\mathrm{F} / \mathrm{m}^{2}\right) . V_{\mathrm{F}_{\text {out. }}}$ is higher than $V_{\text {pos. }}$, then $V_{\mathrm{F}_{\text {out }}}$ becomes $\mathrm{BDV}$.

$$
\begin{aligned}
& \mathrm{BDV}=V_{\mathrm{F}_{\text {out. }}}=\frac{1}{\mathrm{D}} V_{\text {neg. }} \\
& \left(C_{\mathrm{b}}=2.737 \times 10^{-9}, l=\left(100-r_{\mathrm{e}}\right) \times 10^{-3}\right)
\end{aligned}
$$

\section{ESTIMATED BDV AND COMPARISON WITH EXPERIMENTS}

According to the BDV estimation procedure, the surface breakdown voltage was calculated and was compared with the measured values.

\subsection{SPECIFICATIONS OF SAMPLES}

Table 2 shows the specifications of the test samples used in the measurement of the surface breakdown voltage.

The test samples were classified into three groups due to the variable which was one of three parameters of the test sample, $r_{\mathrm{e}}, t$ or $r_{\mathrm{p}}$.

\subsection{AC BDV}

Figure 7 shows the relation between ac breakdown voltage (BDV) and the radius of the embedded electrode, $r_{\mathrm{e}}$. Surface breakdown voltage was measured in the previous study [3] with voltage elevation method. Voltage elevation rate was about $3 \mathrm{kV} / \mathrm{s}$. Measurement was done more than three times for each condition of samples listed in Table 2 and the minimum value was employed as BDV. Although the measured BDV is smaller than the estimated value when $r_{\mathbf{e}}$ is smaller than $25 \mathrm{~mm}$, the measured BDV is almost the same as the estimated value when $r_{\mathrm{e}}$ is larger than $25 \mathrm{~mm}$. The difference in the small $r_{\mathrm{e}}$ region will be explained in DISCUSSION.

Table 2. Specifications of test samples.

\begin{tabular}{c|c|c|c}
\hline $\begin{array}{c}\text { Radius of } \\
\text { the plate } \\
(\mathrm{mm})\end{array}$ & $\begin{array}{c}\text { Thickness of } \\
\text { the plate } \\
(\mathrm{mm})\end{array}$ & $\begin{array}{c}\text { Radius of the } \\
\text { embedded } \\
\text { Electrode }(\mathrm{mm})\end{array}$ & $\begin{array}{c}\text { Divided } \\
\text { voltage } \\
\text { ratio, D }\end{array}$ \\
\hline 100 & 11 & 5 & 1.00 \\
100 & 11 & 15 & 0.66 \\
100 & 11 & 25 & 0.69 \\
100 & 11 & 50 & 0.69 \\
100 & 11 & 70 & 0.69 \\
100 & 9 & 50 & 0.73 \\
100 & 15 & 50 & 0.65 \\
100 & 21 & 50 & 0.61 \\
50 & 11 & 25 & 0.69 \\
250 & 11 & 25 & 0.68 \\
\hline
\end{tabular}

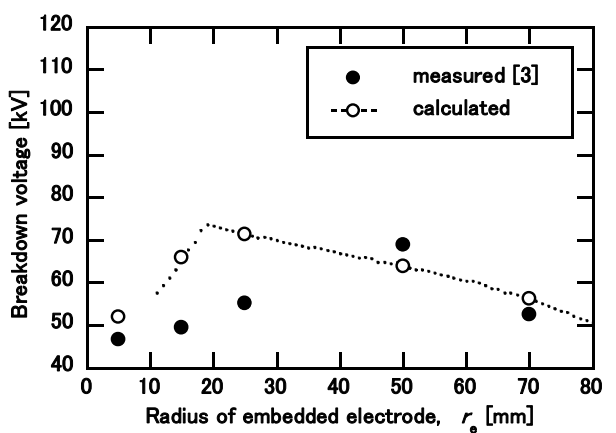

Figure 7. Relation between ac breakdown voltage and the radius of the embedded electrode. $t=11(\mathrm{~mm})$ and $r_{\mathrm{p}}=100(\mathrm{~mm})$.

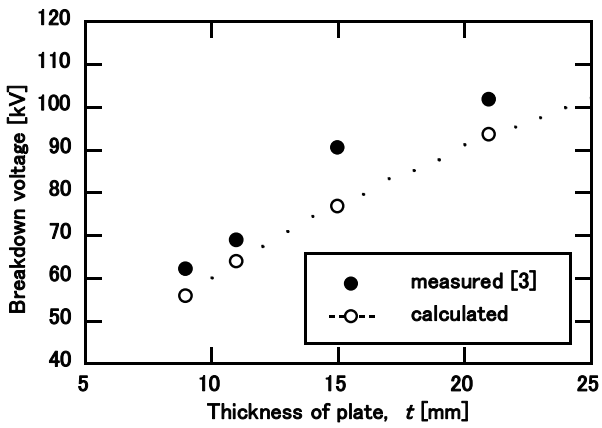

Figure 8. Relation between ac breakdown voltage and the thickness of the plate. $r_{\mathrm{e}}=50(\mathrm{~mm})$ and $r_{\mathrm{p}}=100(\mathrm{~mm})$.

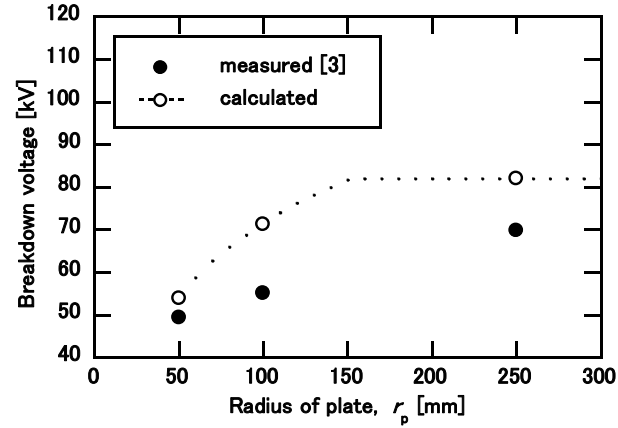

Figure 9. Relation between ac breakdown voltage and the radius of the plate. $r_{\mathrm{e}}=25(\mathrm{~mm})$ and $t=11(\mathrm{~mm})$.

Figure 8 shows the relation between ac $\mathrm{BDV}$ and the thickness of the plate, $t$. There is a good correlation between the measured value and the estimated value. According to the increase of $t$, the distributed electrostatic capacity per unit area decreases and the surface flashover voltage increases. Therefore, the surface breakdown voltage increases with $t$.

Figure 9 shows the relation between ac BDV and the radius of the plate, $r_{\mathrm{p}}$. The estimated value is higher than the measured value. The difference will be explained in DISCUSSION.

\subsection{IMPULSE BDV}

Figure 10 shows the relation between impulse breakdown voltage (BDV) and the radius of the embedded electrode, $r_{\mathrm{e}}$. Surface breakdown voltage was measured in the previous study [3] with $1.2 / 50 \mu$ s impulse voltage and voltage elevation 
method. Voltage elevation was started from around $80 \%$ of the estimated breakdown voltage and the step was about $1 \mathrm{kV}$. Residual charge was removed before each voltage application.

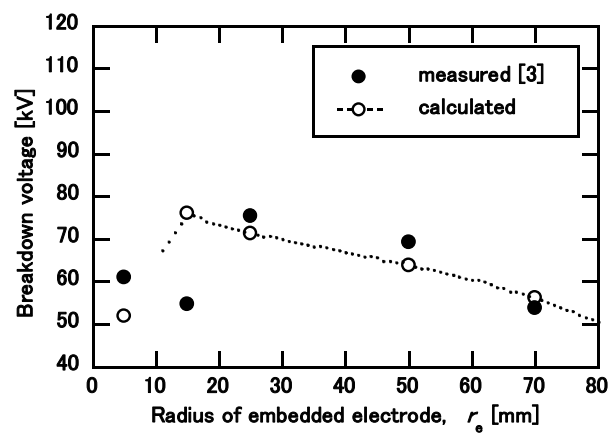

(a) positive

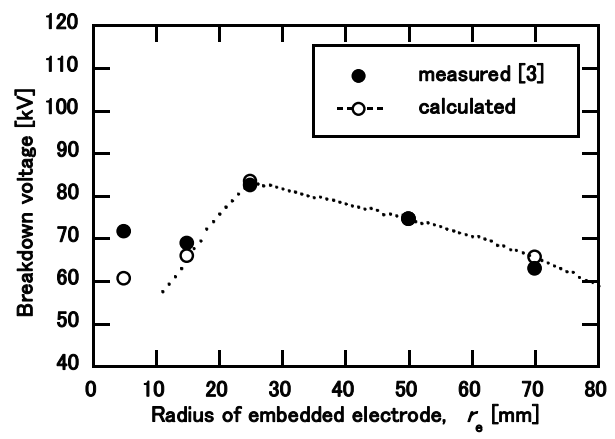

(b) negative

Figure 10. Relation between impulse breakdown voltage and the radius of the embedded electrode. $t=11(\mathrm{~mm})$ and $r_{\mathrm{p}}=100(\mathrm{~mm})$.

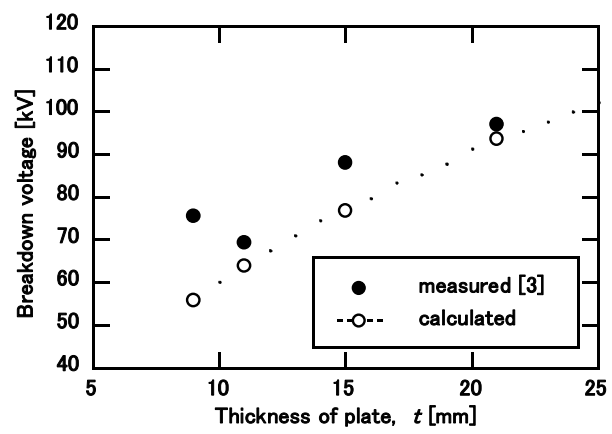

(a) positive

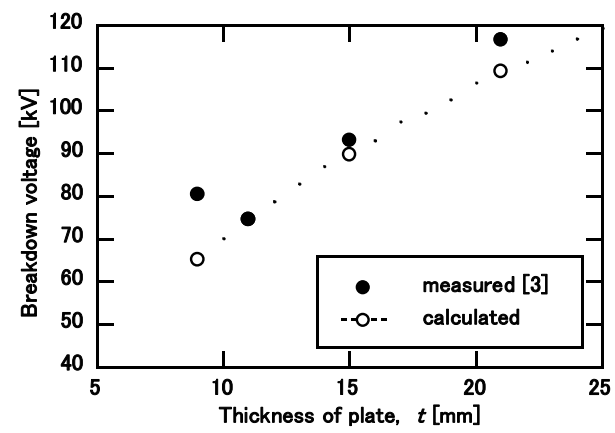

(b) negative

Figure 11. Relation between impulse breakdown voltage and the thickness of the plate. $r_{\mathrm{e}}=50(\mathrm{~mm})$ and $r_{\mathrm{p}}=100(\mathrm{~mm})$.

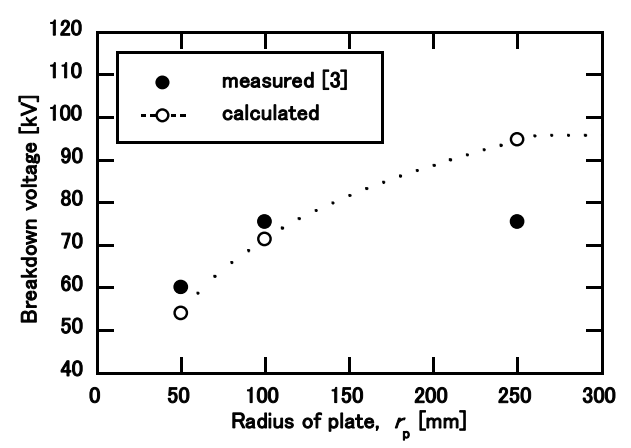

(a) Positive

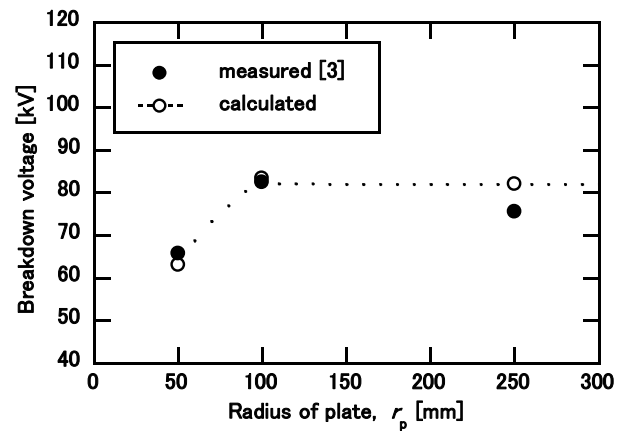

(b) negative

Figure 12. Relation between impulse breakdown voltage and the radius of the plate. $r_{\mathrm{e}}=25(\mathrm{~mm})$ and $t=11(\mathrm{~mm})$.

Measurement was done more than 20 times for each condition of samples listed in Table 2 and the minimum value was employed as BDV.

In both cases of positive and negative impulse application, when $r_{\mathrm{e}}$ is larger than $15 \mathrm{~mm}$ there are good correlation between the measured value and the estimated value. When $r_{\mathrm{e}}$ is $15 \mathrm{~mm}$, the measured value is smaller than the estimated value under positive impulse. The difference will be explained in DISCUSSION.

Figure 11 shows the relation between impulse breakdown voltage (BDV) and the thickness of the plate, $t$. In both cases of positive and negative impulse application, there are relatively good correlations between the estimated value and the measured value.

Figure 12 shows the relation between impulse BDV and the radius of the plate, $r_{\mathrm{p}}$. In both cases, there are good correlations between the estimated value and the measured value except for the large $r_{\mathrm{p}}$ region under positive impulse. The difference in large $r_{\mathrm{p}}$ region is unexpected and further investigation is necessary.

\section{DISCUSSION}

\subsection{PARTICULARITY IN SMALL $r_{e}$}

Figure 13 shows the electric field distribution along the surface of the epoxy resin plate in the small $r_{\mathrm{e}}$ region. The radius of the center rod electrode was $5 \mathrm{~mm}$. Electric field distribution was obtained using Ansoft Maxwell (Ansoft Japan Co., Ltd.) based on finite element method. 


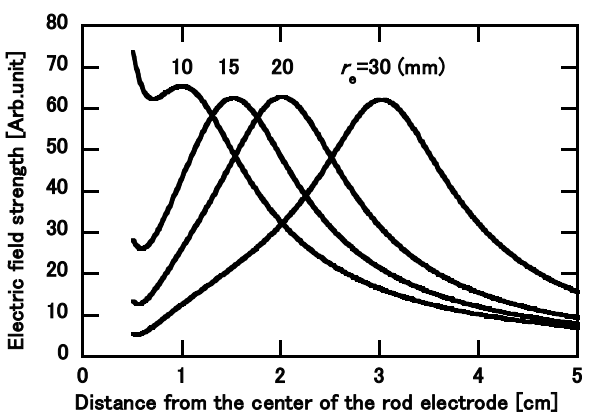

Figure 13. Variation of electric field distribution along the surface of the epoxy resin plate as a parameter of the radius of the embedded electrode. $t=11(\mathrm{~mm})$ and $r_{\mathrm{p}}=100(\mathrm{~mm})$.

When $r_{\mathrm{e}}$ is smaller than $10 \mathrm{~mm}$, the point of the maximum field strength appears on the surface of the center rod electrode. Then, the partial discharge is initiated at the surface of the center rod electrode and it will cover the surface around the center rod electrode easily because the electric field around the center rod electrode is high.

In the case where $r_{\mathrm{e}}=15(\mathrm{~mm})$, the point of the maximum field strength appears close to just above the edge of the embedded electrode, however, the electric field at the surface of the center rod electrode is still high. When the partial discharge is initiated at the point of the maximum field strength, the electric field along the inner surface is enhanced by the existence of the partial discharge. Therefore, the inner surface is also supposed to be covered by the partial discharge easily.

Figure 14 shows the aspects of ac partial discharge when the radii of the embedded electrode are 15 and $25 \mathrm{~mm}$, and the applied voltage is just above PDIV. As shown in Figure 14b, when the radius of the embedded electrode was $15 \mathrm{~mm}$, the partial discharge appeared around the center rod electrode and around the position just above the edge of the embedded electrode, and sometimes covers the inner surface.

When the radius of the embedded electrode was $25 \mathrm{~mm}$, the partial discharge appeared around the position just above the edge of the embedded electrode, as shown in Figure 14c. However, a faint partial discharge can be seen at the surface of the center rod electrode. Therefore, there still be the effect of field enhancement by the partial discharge when the radius of the embedded electrode was $25 \mathrm{~mm}$.

According to this effect, flashover voltage of the inner surface will decrease and the BDV will become lower, in consequence.

\subsection{PARTICULAR CASES OF BDV}

As mentioned above, some particular cases appear when $r_{\mathrm{e}}$ is small.

\section{(1) BDV- $r_{\mathrm{e}}$ characteristics}

In Figure 10, the measured $\mathrm{BDV}$ is smaller than the estimated value in the case where $r_{\mathrm{e}}=15(\mathrm{~mm})$ and the positive impulse application. The reason of the difference is explained by the effect of field enhancement mentioned in 5.1. The surface flashover voltage of inner surface is supposed to

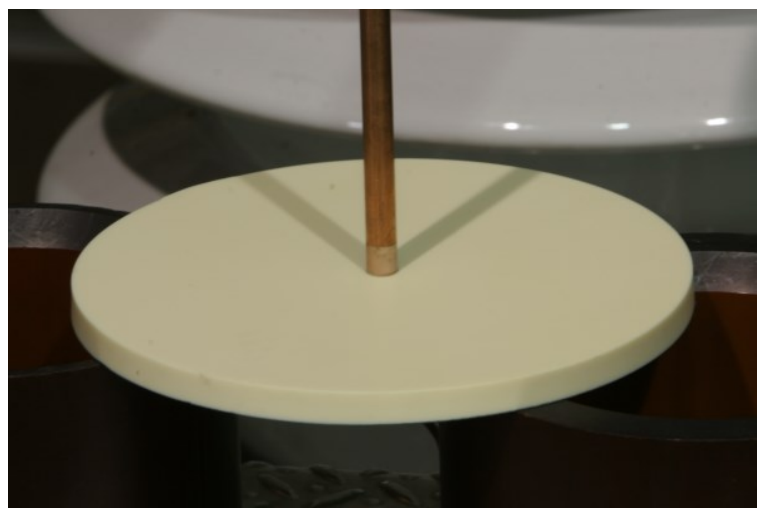

(a) test sample [3]

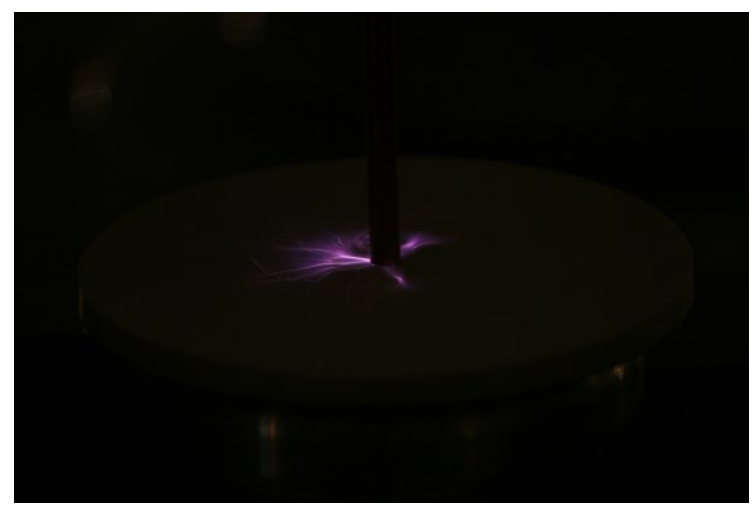

(b) $r_{\mathrm{e}}=15(\mathrm{~mm})$ and the applied voltage was $32 \mathrm{kV}_{\mathrm{rms}}$

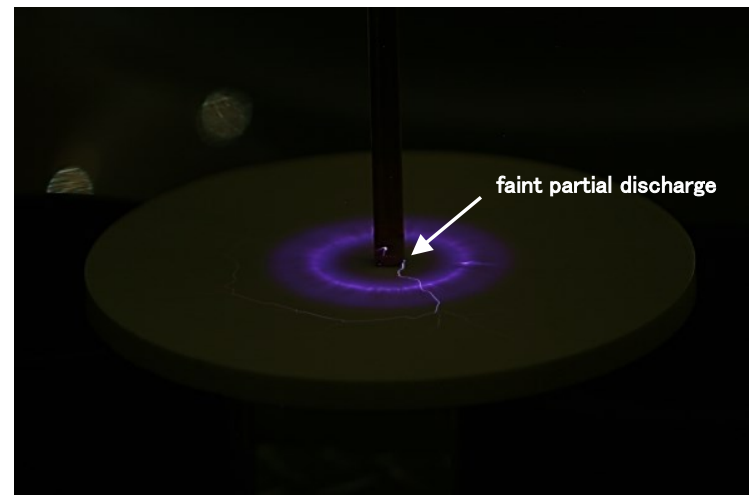

(c) $r_{\mathrm{e}}=25(\mathrm{~mm})$ and the applied voltage was $36 \mathrm{kV}_{\mathrm{rms}}$

Figure 14. Aspect of partial discharge under ac voltage. $t=11(\mathrm{~mm})$ and $r_{\mathrm{p}}=100(\mathrm{~mm})$.

become the same as PDIV. Therefore, measured BDV became smaller than the estimated BDV.

In Figure 7, the measured BDV is smaller than the estimated value when $r_{\mathrm{e}}$ is smaller than $25 \mathrm{~mm}$ under ac voltage application. In the case of ac, the field enhancement is also one of the reason of the difference. Moreover, the effect of the residual charge on the surface discharge propagation is supposed. From the comparison between the measured BDV and the estimated value, the effect must be remarkable on the inner surface of short gap length up to $25 \mathrm{~mm}$.

(2) BDV- $r_{p}$ characteristics

In Figure 9, the measured BDV is smaller than the estimated BDV in the case of ac. In this case, the radius of the embedded electrode was $25 \mathrm{~mm}$, then the effect mentioned 
above was supposed to appear remarkably. BDV will become higher if the radius of the embedded electrode is chosen larger than $25 \mathrm{~mm}$.

\section{CONCLUSION}

Solid insulated switchgear (SIS) has been developed as substitution for medium voltage $\mathrm{SF}_{6}$ gas insulated switchgear. In the solid insulated switchgear, solid/gas insulation system is formed. For further miniaturization and higher stress design, the authors investigated on the surface insulation using the test sample, which was composed of the disc-shaped epoxy resin plate with embedded electrode. In the present study, the surface discharge propagation characteristics were measured with a disc-type epoxy plate and an equation which express the characteristics was obtained. The surface breakdown voltage was estimated based on the obtained equation. The results are summarized as follows.

(1) The characteristics of the flashover voltage along the surface of the disk-type epoxy resin plate is found to be different from the conventional equation.

(2) From the measurement of the surface flashover voltage, an equation which express the characteristics of the flashover voltage was obtained.

(3) The estimation method of the breakdown voltage on the solid/gas insulation with embedded electrode based on the surface flashover voltage, distributed electrostatic capacity of the surface, and the propagation direction and the polarity of the surface discharge was proposed.

(4) The estimated surface breakdown voltage based on the proposed estimation method agrees well with the measured value except for the region where the radius of the embedded electrode is small.

(5) The difference between the estimated value and the measured value in the region where the radius of the embedded electrode is small was supposed to be caused by the effect of field enhancement by the partial discharge and the residual charge.

From the above, the surface breakdown voltage estimation procedure of the solid/gas composite insulation with embedded electrode is established.

\section{REFERENCES}

[1] T. Shioiri, J. Sato, T. Ozaki, O. Sakaguchi, T. Kamikawaji, M. Miyagawa, M. Homma and K. Suzuki, "Insulation Technology for Medium Voltage Solid Insulated Switchgear", Annual Report Conference on Electrical Insulation and Dielectric Phenomena, pp.341$344,2003$.

[2] J. Sato, O. Sakaguchi, N. Makishima, S. Kinoshita, T. Shioiri, T. Yoshida, M. Miyagawa, M. Homma and E. Kaneko, "New Technology for Medium Voltage Solid Insulated Switchgear", Proc. IEEE PES T\&D Conference 2002, Vol.3, pp.1791-1796, 2002.

[3] T. Yamashita, K. Iwanaga, T. Furusato, H. Koreeda, T. Fujishima and J. Sato, "Improvement of Insulation Performance of Solid/Gas Composite Insulation with Embedded Electrode", IEEE Trans. Dielect. Electr. Insul. Vol.23, pp.787-794, 2016.

[4] M. Toepler, "Zür Kenntnis der Gesetze der Gleitfunkenbildung", Ann. Physik, Band 21, pp.193-222, 1906.

[5] M. Toepler, "Über die physikalischen Grundgesetze der in der Isolatorentechnik auftretenden elektrischen Gleiterscheinungen", Arch. Elektrotechnik, Band 10, pp.157-185, 1921.

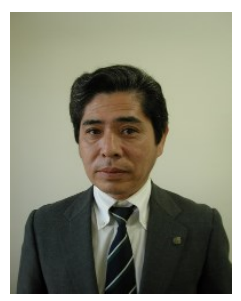

Takahiko Yamashita (M'00) was born in 1957 in Fukuoka, Japan. He received B.E., M.E. and D.E. degrees from Kyushu University in 1980, 1982 and 1985, respectively. He has been working in Nagasaki University, Nagasaki, Japan since 1985. He is a Professor of the Graduate School of Engineering and a Vice President of Nagasaki University. He is a senior member of Institute of Electrical Engineers Japan.

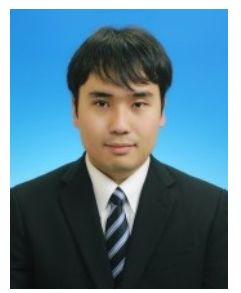

Kazuhisa Iwanaga was born in Nagasaki, Japan, in 1991. He received B.S., degree from Nagasaki University, Nagasaki Japan, in 2014, where he is currently pursuing on M.S. degree with the Graduate School of Engineering. He is mainly engaged in the development of gas/solid composite insulation systems under high stress conditions.

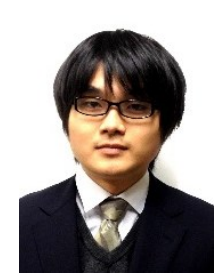

Tomohiro Furusato (M'11) was born in Kagoshima, Japan, in 1988. He received B.S., M.S., and Ph.D. degrees from Kumamoto University, in 2011, 2012, and 2014, respectively. He was with the Japan Society for the Promotion of Science, Kumamoto University, from 2013 to 2014, as a Research Fellow. Since 2014, he has been an Assistant Professor with the Graduate School of Engineering, Nagasaki University. His research interests are pulsed-power, creeping discharge, and discharge phenomena in supercritical fluids.

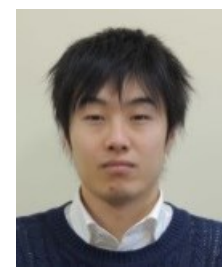

Hiroyuki Koreeda was born in Miyazaki, Japan, 1985. $\mathrm{He}$ received B.S. and M.S. Eng. degrees in electrical engineering from Nagasaki University, Nagasaki Japan, in 2008 and 2010. He was with the Mitsumi Electric Co., Ltd. from 2010 to 2013. Since 2014, he has been working as a technician in Nagasaki University.

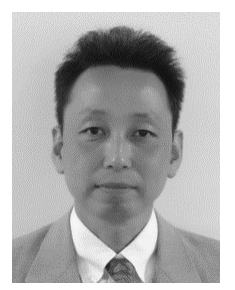

Tomoyuki Fujishima was born in 1966 in Fukuoka, Japan. He received B.E., M.E. and D.E. degrees from Kumamoto University, Japan, in 1989, 1991 and 1994, respectively. $\mathrm{He}$ has been working in Nagasaki University since 1994 and is an Associate Professor of the Graduate School of Engineering. His research interests are ozone generation, ozonic environment application and lightning protection.

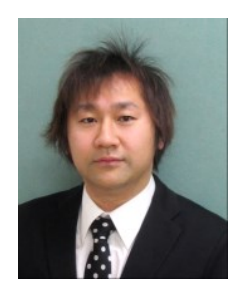

Naoki Asari was born in 1980 in Japan. He received B.S. and M.S. in electrical engineering from Saitama University in 2003 and 2005, respectively. He joined Toshiba Corporation in 2005. He is now with the switchgear and sensing system technology group. He has been engaged in research on insulation technology for switchgear and vacuum interrupter.

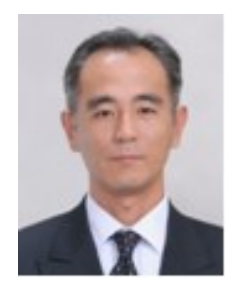

Junichi Sato was born in 1967 in Fukuoka, Japan. He received B.E., M.E. and D.E. degrees from Nagasaki University in 1990, 1992 and 2011, respectively. He has been working in Toshiba Corporation, Tokyo, Japan since 1992. He belongs to the power and industrial systems research and development center, Toshiba Corporation. His research interests are solid and solid/gas composite insulation systems, vacuum interrupter and solid insulation switchgear. 